\title{
Observations of the Brackett decrement in the Class I source HH100 IR ${ }^{\star}$
}

\author{
B. Nisini ${ }^{1}$, S. Antoniucci ${ }^{2,1}$, and T. Giannini ${ }^{1}$ \\ 1 INAF-Osservatorio Astronomico di Roma, 00040 Monteporzio Catone, Italy \\ 2 Università degli Studi “Tor Vergata”, via della Ricerca Scientifica 1, 00133 Roma, Italy
}

Received 24 September 2003 / Accepted 3 March 2004

\begin{abstract}
The Brackett decrement in the Class I source HH100 IR has been observed and analyzed to set constraints on the origin of the IR HI emission in this young object. We have used both low resolution $(R \sim 800)$ observations of the Brackett lines from $\mathrm{Br} \gamma$ to $\mathrm{Br} 24$, and medium resolution $(R \sim 9000)$ spectra of the $\mathrm{Br} \gamma, \mathrm{Br} 12$ and $\mathrm{Br} 13$ lines. The dereddened fluxes indicates that the lines remain moderately thick up to high quantum numbers. Moreover, the profiles of the three lines observed in medium resolution are all broad and nearly symmetric, with a trend for the lines at high $n$-number to be narrower than the Br $\gamma$ line. With the assumption that the three lines have different optical depths and consequently trace zones at different physical depths, we interprete the observed profiles as evidence that the ionized gas velocity in the $\mathrm{HI}$ emitting region is increasing as we move outwards, as expected in an accelerating wind more than in an infalling gas.

We have modelled the observed line ratios and velocities with a simplified model for the HI excitation from a circumstellar gas with a velocity law $V=V_{0}+\left(V_{\max }-V_{0}\right)\left(1-\left(r_{i} / r\right)^{\alpha}\right)$. Such a comparison indicates that the observations are consistent with the emission coming from a very compact region of 4-6 $R_{\odot}$, where the gas has been already accelerated to velocities of the order of $200 \mathrm{~km} \mathrm{~s}^{-1}$, with an associated mass flow rate of the ionized component of the order of $10^{-7} M_{\odot} \mathrm{yr}^{-1}$. This implies that the observed lines should originate either from a stellar wind or from the inner part of a disk wind, providing that the disk inner truncation radius is close to the stellar surface. It is also expected that the gas ionization fraction is relatively high as testified by the high rate of ionized mass loss derived. Our analysis, however, does not resolve the problem of how to reproduce the observed symmetrical line profiles, which at present are apparently difficult to model by both wind and accretion models. This probably points to the fact that the real situation is more complicated than described in the simple model presented here.
\end{abstract}

Key words. line: formation - stars: circumstellar matter - stars: individual: HH100-IR - infrared: stars - stars: formation stars: winds, outflows

\section{Introduction}

The emission from hydrogen recombination lines represents the most direct manifestation of circumstellar activity in young stars. Lines from the Balmer series have been the main spectroscopic tool for identifying classical T Tauri and Herbig AeBe stars. In spite of the large amount of observational data, however, the HI lines are so easily excited that it is very difficult to clearly define their origin. Originally, they were interpreted as being excited in circumstellar ionized winds, on the basis of the often observed P Cygni profile exhibited by the $\mathrm{H} \alpha$ line (Hartmann et al. 1990; Calvet et al. 1992). More recently, such an interpretation has been challanged due to the variety of the observed Balmer line profiles (e.g. Edwards et al. 1994; Reipurth et al. 1996) that are difficult to reproduce by means of wind models, and the HI emission has been

\footnotetext{
Send offprint requests to: $\mathrm{B}$. Nisini, e-mail: nisini@mporzio.astro.it

* Based on observations collected at the European Southern Observatory, Chile (ESO N.069.C-0269).
}

interpreted also in the framework of magnetospheric accretion models (Calvet \& Hartman 1992; Muzerolle et al. 1998a). Studies of HI lines in pre-main sequence stars have been extended also in the near and mid IR (Folha \& Emerson 2001; Benedettini et al. 1998; Nisini et al. 1995; Natta et al. 1988). While the IR HI line ratios are well reproduced by wind models, the same models apparently fail to explain the observed profiles of the $\mathrm{Br} \gamma$ and $\mathrm{Pa} \beta$ lines. On the other hand, magnetospheric accretion models were able to reproduce the $\mathrm{Br} \gamma$ profiles showing redshifted absorption components (Muzerolle et al. 1998b) but not all the variety of profiles exhibited by T Tauri stars (Folha \& Emerson 2001). Finally, very recently, spectro-astrometric observations of the $\mathrm{Pa} \beta$ line in four T Tauri stars, have indicated that the emission originates in the outflowing material (Whelan et al. 2004).

While there is ample literature on the origin and observations of HI lines in T Tauri and Herbig stars, the interpretation of HI lines in more embedded Class I sources, for which optical observations of the Balmer series are impeded, is much less defined. Observations of spectrally-resolved $\mathrm{HI}$ lines in 


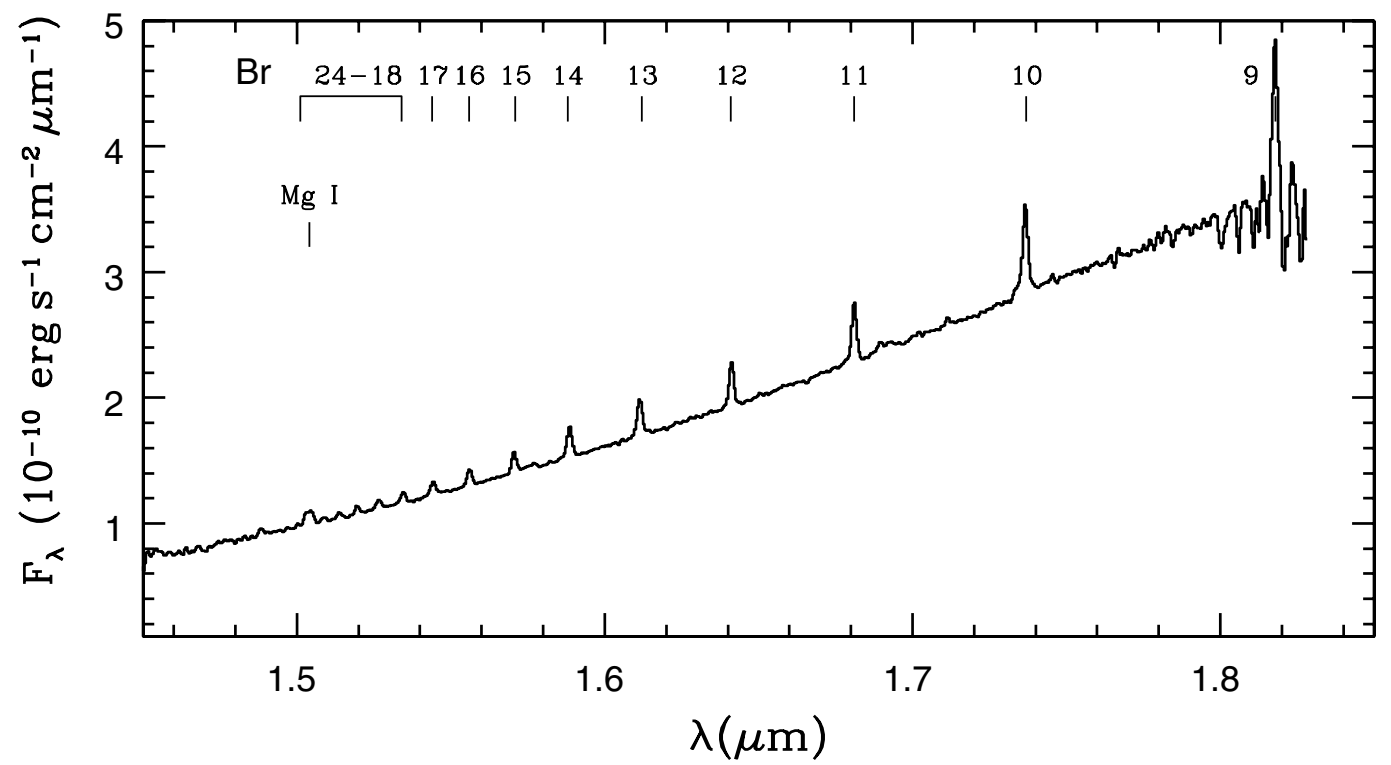

Fig. 1. Low resolution spectrum in the $H$ band of HH100 IR, showing the emission lines from the Brackett series.

low-mass Class I sources so far have been limited to the Br $\gamma$ line (Najita et al. 1996; Davis et al. 2001). At variance with $\mathrm{H} \alpha$ in $\mathrm{T}$ Tauri stars, the profiles of the $\mathrm{Br} \gamma$ line appear more symmetric, with no evidence of the typical P Cygni signature. On this basis Najita et al. (1996) argue against a wind origin, while in the framework of magnetospheric accretion models, Muzerolle et al. (1998b) use the Br $\gamma$ luminosity in a sample of Class I sources to infer the mass accretion rates.

To better constrain the emission region and the mechanism for the excitation of HI lines in Class I sources it is necessary to obtain a larger observational set, including both information on line profiles and flux ratios among different lines. The aim of this work is to start such an analysis using both the ratios from lines in the Brackett series (the Brackett decrement) and high resolution spectroscopy of different Brackett lines. The source investigated here is HH100 IR, a low mass ( $L_{\text {bol }} \sim 10 L_{\odot}$, Wilking et al. 1992) Class I object located in the R CrA star forming core $(D=130 \mathrm{pc}$, Marraco \& Rydgren 1981). This source already has been recognized as an embedded protostar with high circumstellar activity on the basis of its near IR specrum (Greene \& Lada 1996; Nisini et al. 2004), and as also suggested by the fact that it is the driving source of an Herbig Haro object. It therefore represents a suitable test case to perform a detailed analysis of its $\mathrm{HI}$ emission.

\section{Observations}

The observations were performed with the ISAAC spectrometer, at the VLT UT1 telescope, during 12 and 13 July 2002. In the low resolution mode observations we employed a 0.6 slit to acquire spectra covering the $H$ and $K$ bands at $R \sim 800$. In the medium resolution mode, two spectral segments were acquired centered at $1.629 \mu \mathrm{m}$ and at $2.161 \mu \mathrm{m}$ and covering about $0.6 \mu \mathrm{m}$ each. For these observations a 0.3 slit was used, giving a resolution of $\sim 8000$ and 9000 at 2.1 and $1.6 \mu \mathrm{m}$, respectively. For both low and medium resolution observations, spectra of a standard star of B spectral type were obtained at an airmass similar to the scientific spectra, to correct for telluric absorption and obtain flux calibrations. The telluric spectra were carefully cleaned of any intrinsic HI absorption feature before being used. Wavelength calibrations were performed both using a xenon lamp spectrum taken at the end of the night, and refined each time on the $\mathrm{OH}$ sky lines observed in the spectra. This procedure leads to a wavelength calibration error of $\sim 0.1 \AA$ (i.e. about $1-2 \mathrm{~km} \mathrm{~s}^{-1}$ ).

In the low resolution spectra we detected the $\operatorname{Br} \gamma$ at $2.166 \mu \mathrm{m}$ in the $K$ band spectrum, and lines from the higher levels of the Brackett series, from $\mathrm{Br} 9$ up to $\mathrm{Br} 24$, in the $H$ band (Fig. 1 and Table 1). The Br23 line, which shows a line flux much higher than the adjacent lines, is blended with a feature of $\mathrm{Mg}$ I. The medium resolution spectra covered the $\operatorname{Br} \gamma$, the $\operatorname{Br} 12(1.6113 \mu \mathrm{m})$ and the $\operatorname{Br} 13(1.6411 \mu \mathrm{m})$ lines, which all appear resolved. Figure 2 shows the profiles of the lines, while in Table 2 we report the velocity information $\left(V_{\mathrm{LSR}}\right.$ and $\Delta V(F W H M))$ derived through a Gaussian fit. We estimate an error in the line width determination that is between 5 and $10 \mathrm{~km} \mathrm{~s}^{-1}$, based on the difference between the FWHM of the Gaussian fit with that directly measured with respect to the observed peak. The $V_{\mathrm{LSR}}$ velocities are corrected for the systemic velocity of the $\mathrm{R} \mathrm{CrA}$ cloud taken as $5.8 \mathrm{~km} \mathrm{~s}^{-1}$ (Harju et al. 1993).

\section{Line ratios}

The observations of a large number of HI lines from the Brackett series allow a detailed study of the decrement of this series, from which information on the line excitation can be derived. In normal HII regions, the decrement is actually used to derive the extinction through the emitting region, assuming that Case B recombination holds (Hummer \& Storey 1987). However, the excitation of NIR HI lines in Young Stellar Object (YSO) envelopes usually cannot be reconciled with a standard Case B HII region since, due to the high densities of the source circumstellar gas, the lines may remain optically thick up to 
Table 1. Low resolution observations.

\begin{tabular}{cccc}
\hline \hline & & \multicolumn{2}{c}{ HH100 IR } \\
$\lambda(\mu \mathrm{m})$ & Line & Flux $^{a}$ & $\Delta F^{a}$ \\
\hline 1.501 & Br 24 & 3.5 & 0.3 \\
1.504 & Br 23 + Mg I & 20.1 & 0.7 \\
1.509 & Br 22 & 6.1 & 0.7 \\
1.514 & $\operatorname{Br} 21$ & 7.3 & 0.6 \\
1.519 & $\operatorname{Br} 20$ & 10.5 & 0.6 \\
1.527 & $\operatorname{Br} 19$ & 15.5 & 1.0 \\
1.534 & $\operatorname{Br} 18$ & 16.2 & 0.9 \\
1.544 & $\operatorname{Br} 17$ & 23 & 1 \\
1.556 & $\operatorname{Br} 16$ & 24.8 & 0.9 \\
1.571 & $\operatorname{Br} 15$ & 32.5 & 1.0 \\
1.588 & $\operatorname{Br} 14$ & 51 & 2 \\
1.611 & $\operatorname{Br} 13$ & 64 & 2 \\
1.641 & $\operatorname{Br} 12$ & 66 & 2 \\
1.681 & $\operatorname{Br} 11$ & 94 & 3 \\
1.737 & $\operatorname{Br} 10$ & 155 & 5 \\
1.818 & $\operatorname{Br} 9$ & 229 & 26 \\
2.166 & $\operatorname{Br} \gamma$ & 513 & 17 \\
\hline
\end{tabular}

${ }^{a}$ Fluxes and their errors are expressed in $10^{-15} \mathrm{erg} \mathrm{cm}^{-2} \mathrm{~s}^{-1}$.

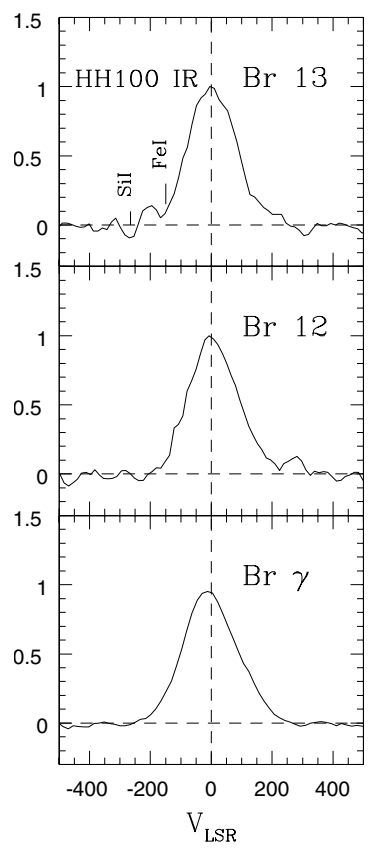

Fig. 2. Resolved spectra of the $\mathrm{Br} \gamma, \mathrm{Br} 12$ and $\mathrm{Br} 13$ lines in HH100 IR. The underlying continuum of each spectrum has been subtracted and each line is normalized to its peak intensity. The $V_{\mathrm{LSR}}$ is relative to the stellar velocity (i.e. the velocity of the associated molecular cloud has been subtracted).

high- $n$ levels. Therefore, to proceed with a correct analysis of the observed line ratios we first need to correct the observed lines for the reddening. Estimates on the visual extinction for HH100 IR, based on the optical depth of both the silicate feature at $9.1 \mu \mathrm{m}$ and the $3 \mu \mathrm{m}$ ice band absorption feature, suggest a value around $25 \mathrm{mag}$ (Whittet et al. 1996). Such a value is
Table 2. High resolution observations.

\begin{tabular}{cccc}
\hline \hline & & \multicolumn{2}{c}{ HH100 IR } \\
$\lambda$ & Line & $\begin{array}{c}V_{\text {LSR }}^{a} \\
\mathrm{~km} \mathrm{~s}^{-1}\end{array}$ & $\begin{array}{c}\Delta V \\
\mathrm{~km} \mathrm{~s}^{-1}\end{array}$ \\
\hline$\mu \mathrm{m})$ & & -2 & 184 \\
1.6114 & $\mathrm{Br} 13$ & -3 & 190 \\
1.6412 & $\mathrm{Br} 12$ & -17 & 224 \\
2.1661 & $\mathrm{Br} \gamma$ & -17 \\
\hline
\end{tabular}

${ }^{a} V_{\mathrm{LSR}}$ corrected for the velocity of the R CrA cloud $\left(5.8 \mathrm{~km} \mathrm{~s}^{-1}\right.$, Hariu et al. 1993).

not affected by the IR variability of HH100 IR (Molinari et al. 1994) since Graham (1998) found no variability in the $3 \mu \mathrm{m}$ ice band over a period of $7 \mathrm{yr}$. In addition, an $A_{\mathrm{v}}$ between 20 and 30 mag has been also estimated from the comparison among the intrinsic and observed $(H-K)$ colors, assuming the stellar spectral type derived from the photospheric features detected in the IR spectrum of the source (Nisini et al. 2004). Therefore a value of $A_{\mathrm{V}}=25 \mathrm{mag}$, together with the reddening law by Rieke \& Lebofsky (1985), have been adopted to correct the observed fluxes for the reddening. Figure 3 shows the ratios of the different Brackett lines with respect to the $\mathrm{Br} \gamma$ line intensity. In the same figure the ratios expected for a Case B recombination are also plotted for the extreme cases of $T=6000 \mathrm{~K}, n=$ $10^{4} \mathrm{~cm}^{-3}$ and $T=10000 \mathrm{~K}, n=10^{6} \mathrm{~cm}^{-3}$, taken from Storey $\&$ Hummer (1995). The observed ratios are always higher than the Case B values, which indicates that the Brackett lines remain optically thick up to high values of the $n$ number. The displacement from the Case B curves could be due to a wrong extinction value assumed, but an $A_{\mathrm{V}}$ value as low as $\sim 5 \mathrm{mag}$ would be needed to reconcile the observations with the Case B recombination.

In the figure, we also show the Brackett decrement in the case of emission from optically thick $(\tau \gg 1)$ lines originating from a region of fixed radius at $T=10000 \mathrm{~K}$. In this extreme case, lines from high $n$-number remain always brighter than the $\mathrm{Br} \gamma$. It is evident from this figure that the observed ratios lie in an intermediate situation between these two extreme cases. Since for lines of a given series $\tau \propto \lambda$, the optical depth also decreases as the $n$-number increases. As a consequence, assuming that each line originates from a surface at which $\tau \sim$ 1 , it is expected that different lines trace a different emitting region whose size decreases as the $n$-number increases (see e.g. discussion in Benedettini et al. 1999).

\section{Line profiles}

The profile of the $\mathrm{Br} \gamma$ line in HH100 IR is broad (FWHM $\gtrsim$ $200 \mathrm{~km} \mathrm{~s}^{-1}$ ) and nearly symmetric. This profile is indeed similar to those observed from other Class I sources (Najita et al. 1996; Davis et al. 2001) and from many T Tauri objects (Folha \& Emerson 2001). These previous studies also showed that the Br $\gamma$ line peaks are often blueshifted, as observed also in our case, where a $V_{\mathrm{LSR}}$ of $-14 \mathrm{~km} \mathrm{~s}^{-1}$ has been measured. Such blue-shifted symmetrical profiles are difficult to reproduce either by wind models and by magnetospheric accretion 


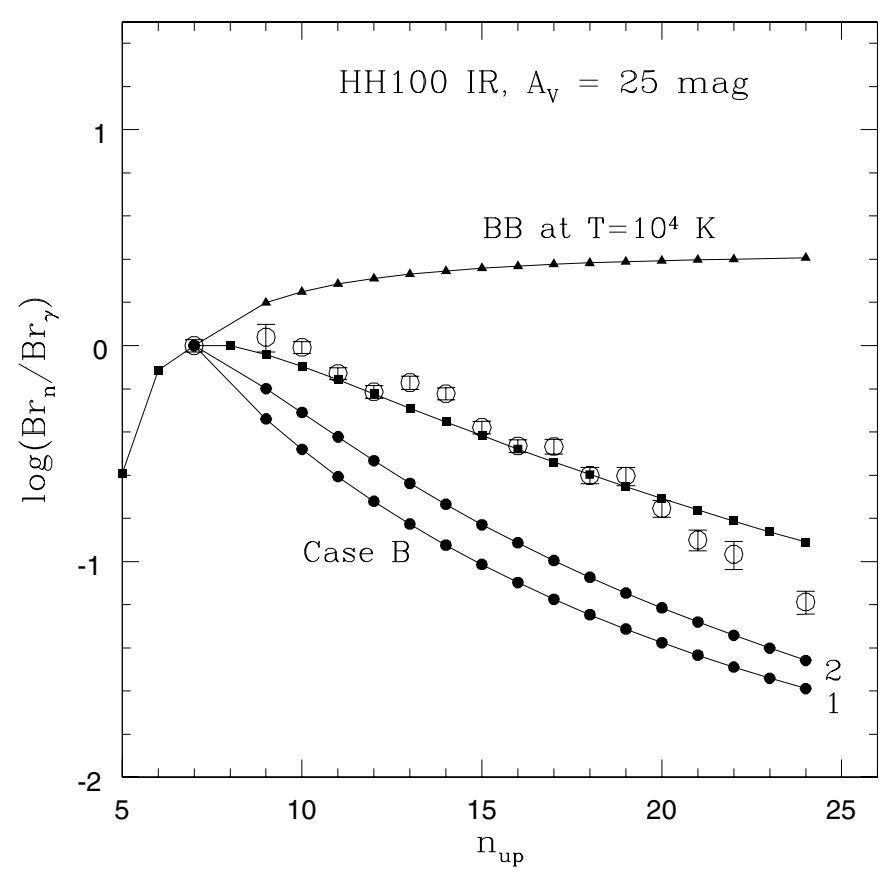

models. Wind models generally predict red-shifted peaks and P-Cygni absorption features. Most of the profile calculations are however done for the Balmer lines (e.g. Calvet et al. 1992), while predictions for NIR lines are seldom found. The $\mathrm{Br} \gamma$ and $\operatorname{Br} \alpha$ profiles derived from Hartmann et al. (1990) in their magnetically driven wind model, do indeed lack P-Cygni absorption features and are more symmetric, with a FWHM of the order of $200 \mathrm{~km} \mathrm{~s}^{-1}$, comparable to the values observed in Class I sources, but they still present pronounced asymmetries and they are mostly red-shifted. In the case of wind models, a blue-shifted centroid may result from the occultation of the redshifted part of the profile by an optically thick circumstellar disk, as is observed in optical forbidden lines (e.g. Edwards et al. 1987). Such an effect however should be accompanied by a significant asymmetry in the redshifted side of the profile which apparently is lacking here. Magnetospheric accretion models, on the other hand, tend to predict profiles with blue-shifted peaks, as in our case, but where such a blue-shift centroid is the result of strong asymmetries caused by the suppression of the redshifted part of the profile (e.g. Muzerolle et al. 1998a). Recently, Muzerolle et al. (2001) provided more refined line profile models, including line damping due to different broading mechanisms, which have the net effect of filling in the red-shifted absorption component causing a much more symmetric and centrally peaked profile. This effect is however particularly important only for the $\mathrm{H} \alpha$ line and becomes less significant for the higher Balmer and near IR lines. More symmetric profiles of the $\mathrm{Br} \gamma$ may be predicted at privileged inclination angles of the accretion disk (Muzerolle et al. 1998a), but even in the extreme situation of $i \sim 70^{\circ}$ the predicted red-shifted asymmetries should be still recognizable in our high $\mathrm{S} / \mathrm{N}$ spectrum. Finally, the absorption of the redshifted side of the profile also cause the FWHM line width to narrow significantly, and indeed the $\mathrm{Br} \gamma$ profiles shown in Muzerolle et al. (1998a, 2001) always have a FWHM of about $100 \mathrm{~km} \mathrm{~s}^{-1}$ or less, thus much narrower than the values
Fig. 3. Ratio of the Brackett lines with respect to the $\mathrm{Br} \gamma$ plotted as a function of the upper quantum number. Open circles are the ratios measured on HH100 IR, dereddened by a visual extinction of 25 mag. The two curves indicated with filled dots are the ratios expected from Case B recombination (Hummer \& Storey 1987) assuming 1- $T=6000 \mathrm{~K}, n=$ $10^{4} \mathrm{~cm}^{-3}, 2-T=10000 \mathrm{~K}, n=10^{6} \mathrm{~cm}^{-3}$. Triangles are the ratios expected in case of emission from an optically thick region at $T=10000 \mathrm{~K}$. The squares represent our best fit model to the observed points assuming a spherically symmetric model with an accelerating velocity law. The parameters for this model are the following (see text for the parameters definition): $r_{0}=3 R_{*}, \dot{M}_{\text {ion }}=$ $2 \times 10^{-7} M_{\odot} \mathrm{yr}^{-1}, V_{0}=30 \mathrm{~km} \mathrm{~s}^{-1}, V_{\max }=$ $230 \mathrm{~km} \mathrm{~s}^{-1}$ and $\alpha=4$.

we observe. Such a discrepancy between the FWHM predicted by the magnetospheric accretion models and the observed wider $\mathrm{Br} \gamma$ line-widths was also pointed out by Folha \& Emerson (2001) for their sample of T Tauri observations. Since the line width depends on the infall velocity field in the accretion flow, which in turn is a function of the source gravitational potential energy, such a width could be larger in sources with a mass greater than the canonical value assumed by the Muzerolle et al. models, i.e. $0.5 M_{\odot}$. In the specific case of HH100 IR, a stellar mass of $0.4 M_{\odot}$ has been estimated (Nisini et al. 2004), so in principle line widths even narrower than those predicted by Muzerolle et al. should be expected.

On the basis of these considerations, we believe that at present both the existing wind and accretion models still fail in reproducing the observed HI near-IR symmetric profiles, and therefore it is not possible to favor one of these two excitation mechanisms on the basis of profiles alone.

The profiles of the observed Br12 and 13 lines also appear broad but with $F W H M$ velocities smaller than the $\mathrm{Br} \gamma$ line. These profiles are also rather symmetric: two apparently blueshifted absorptions in the Br13 line can be ascribed to the presence of two photospheric absorption features, as indicated in Fig. 2. If we correct for these absorption features, the Br13 linewidth becomes $\sim 190 \mathrm{~km} \mathrm{~s}^{-1}$, i.e. more similar to the Br12 line and still significantly lower than the $\mathrm{Br} \gamma$ line-width. It seems therefore to be a trend for the $F W H M$ velocity to decrease with higher quantum numbers. We have seen that the observed Brackett lines are thick and have different optical depths, thus they trace zones at different physical depths in the emitting region. Since moreover the optical depth decreases with increasing upper quantum number (i.e. with decreasing wavelength), it is expected that high- $n$ lines trace regions more internal than the $\operatorname{Br} \gamma$ line. Therefore if we assume that the observed line widths measure the maximum velocity attained at the emitting region surface, the observed trend would imply that the velocity is increasing going outwards, as expected, e.g., 
in an accelerating wind. With the assumption that such a velocity trend can be ascribed to the gas kinematical motion alone, then the different observed linewidths are incompatible with a velocity law due to any accretion process or Keplerian rotation (such as $v \propto r^{-1 / 2}$ ) where the velocity is decreasing with the distance from the central object. Emission from a flattened geometry viewed at different inclination angles cannot produce changes in this trend. Muzerolle et al. (2001), on the other hand, presented evidence that other line broadening effects, radiative and Stark broadening in particular, may dominate the line-width in some circumstances, an effect which is particularly important for the $\mathrm{H} \alpha$ line. However, the Muzerolle et al. (2001) models show that these broadening effects become negligible for near IR lines, and hence the line-widths of the Brackett lines are more likely to reflect the dynamical properties of the gas in the emitting region than other broadening effects.

A rough estimate of the size of the emitting region can be given assuming that the flux in the different lines is given by a blackbody emission at $T \sim 10000 \mathrm{~K}$, i.e.:

$F_{\lambda}=\pi \frac{R_{\tau=1}^{2}}{D^{2}} B_{\lambda}(\lambda, T) \lambda \frac{\Delta V}{c}$.

Taking as $\Delta V$ the widths of the observed lines and a distance of $130 \mathrm{pc}$, we derive that the $\mathrm{Br} \gamma$ emission size is of the order of $4 \times 10^{11} \mathrm{~cm}$ while the $\mathrm{Br} 12,13$ emission sizes are of the order of $2 \times 10^{11} \mathrm{~cm}$. This indicates that the HI lines originate from a region of only $4-6 R_{\odot}$, thus very close to the protostellar photosphere, which is expected to be a few solar radii in diameter. Since the emitting region is not vey large, the central source may produce an occultation of the redshifted part of the flow. A simple way to check the amount of this occultation is through geometrical considerations. The emission originates from the surface at which $\tau \sim 1$, thus the amount of flux not reaching the observer due to the central star can be estimated as the ratio between the emission region surface and the source projected area. This estimate shows that only $\sim 5 \%$ of the total line emission would be hidden by the central source, and thus difficult to detect although it should mainly affect the line redshifted wing. An occultation from a circumstellar disk should in fact produce a more severe effect on the line profile. Such an effect is not observed also in HI lines from T Tauri stars while it is often present in other optical forbidden lines such as [OI] (e.g. Edwards et al. 1987; Takami et al. 2001). This has been interpreted as evidence for the presence of internal disk holes allowing the redshifted part of lines coming from compact regions to reach the observer while obscuring that of the forbidden lines, originating from more external and lower density regions (Takami et al. 2001).

\section{Comparison with models of ionized envelopes}

There are very few models in the literature treating in detail (i.e. with a full radiative transfer treatment and taking into account geometrical effects) the excitation of HI IR lines and providing predictions for both flux ratios and line profiles. Natta et al. (1998) provide $\mathrm{Pa} \beta, \operatorname{Br} \alpha$ and $\mathrm{Br} \gamma$ intensities of T Tauri stars in the framework of a partially ionized wind model but without any prediction of line profiles. Muzerolle et al. (1998a), on the other hand, compute the Bry profile expected from their magnetospheric accretion model, but the line fluxes are derived only for $\mathrm{Pa} \beta$ and $\mathrm{Br} \gamma$ lines.

Giving the difficulty of comparing our data with specific models, we have adopted a more general approach, considering a simple model of an ionized envelope in which the gas velocity follows a general law of the type $V=V_{0}+\left(V_{\max }-\right.$ $\left.V_{0}\right)\left(1-\left(r_{i} / r\right)^{\alpha}\right)$, which assumes that the gas is accelerated at a maximum velocity $V_{\max }$ at a distance that depends on the parameter $\alpha$. The ionized envelope extends from an initial radius $r_{i}$ to an outer radius $r_{0}$. Inside this region, the electron density follows from the continuity equation and it is equal to $n_{\mathrm{e}}=\dot{M} x_{\mathrm{e}} / 4 \pi m_{\mathrm{H}} r^{2} V(r)$, where $x_{\mathrm{e}}$ is the fractional ionization and $\dot{M}$ is the rate of mass flow inside the region. The radiation transfer has been treated in the Sobolev approximation assuming the gas in LTE and following the formalism described in Nisini et al. (1995), where a discussion about the limitations of the adopted assumptions is also given.

We have tuned the input parameters in such a way as to reproduce the line ratios, line fluxes and the observed line widths with the assumption that the FWHM traces the maximum velocity attained in the line emitting region. This last constraint is equivalent to reproducing the emitting region sizes as estimated in the previous section.

In this framework, the optical depth at line center, which is the main parameter affecting the line decrement, is given, as a function of the distance from the initial radius $r_{i}$, by:

$\tau=\frac{K_{v} c}{v r_{i}^{3}}\left(\frac{\dot{M} x_{\mathrm{e}}}{4 \pi m_{\mathrm{H}}}\right)^{2} V(r)^{-3}$

where $K_{v}$ is the line absorption coefficient and $v$ the line center frequency. From this expression, we see that the optical depth is strongly affected by the adopted velocity law, the initial radius $r_{i}$ and the rate of ionized gas flow $\dot{M}_{\text {ion }}=\dot{M} x_{\mathrm{e}}$. We have chosen that the emitting region begins at the protostellar radius $R_{*}$, assumed equal to $3 R_{\odot}$. This is the predicted radius of protostellar objects and it is consistent with the derived value of (3.5 \pm 0.7$) R_{\odot}$ estimated from the inferred spectral type and stellar luminosity (Nisini et al. 2004).

With this condition, to maintain the lines optically thick up to high- $n$ values, the $\dot{M}_{\text {ion }}$ value needs to be sufficently high, e.g. larger than $10^{-7} M_{\odot} \mathrm{yr}^{-1}$. At the same time, the absolute line fluxes are also sensitive, in addition to $\dot{M}_{\text {ion }}$, to the gas temperature and the emitting region size. Assuming $T=$ $8000 \mathrm{~K}$, we find that $r_{0}$ values of a few stellar radii are needed to mantain the observed line fluxes close to the observed absolute values. Colder emission may be consistent with slightly larger emission regions, which however cannot exceed $\sim 8 R_{*}$ for $T \gtrsim 4000 \mathrm{~K}$.

In Fig. 3 we show the predicted decrement in our best fit model with the following parameters: $\dot{M} x_{\mathrm{e}}=2 \times$ $10^{-7} M_{\odot} \mathrm{yr}^{-1}, r_{0}=3 R_{*}, V_{0}=30 \mathrm{~km} \mathrm{~s}^{-1}, V_{\max }=230 \mathrm{~km} \mathrm{~s}^{-1}$ and $\alpha=4$. Such a model also reproduces fairly well the dereddened flux of the Br $\gamma$ line (e.g. $5.9 \times 10^{-12} \mathrm{erg} \mathrm{cm}^{-2} \mathrm{~s}^{-1}$ vs.

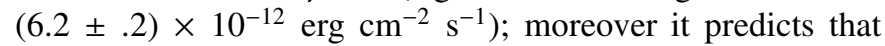
the maximum velocities corresponding to the radii where the 
$\operatorname{Br} \gamma, \operatorname{Br} 12$ and $\operatorname{Br} 13$ lines attain $\tau=1$ are 220, 190 and $180 \mathrm{~km} \mathrm{~s}^{-1}$ respectively, also in agreement with the observed line widths. The model however overestimates the emission from high $n_{\text {up }}$ numbers with respect to the observed values. The high $n_{\text {up }}$ lines originate from inner regions where, according to the adopted velocity law, the speed is still low; consequently, the expected optical depth of these lines is mantained high despite their higher frequency, according to the relationship (2). A better fit to the data can be obtained by assuming a different velocity law with a much higher initial velocity $\left(\sim 180 \mathrm{~km} \mathrm{~s}^{-1}\right)$, which however seems unphysical implying that the ionized gas is flowing from the stellar surface already at a velocity close to the escape speed.

In summary, and considering the oversemplification of the adopted model, we can reasonably conclude that the different observing features can be accounted for by an ionized gas, emitted in a region of a few stellar radii close to the source where the gas is already accelerated at about $200 \mathrm{~km} \mathrm{~s}^{-1}$ and it is expanding, after an initial steep acceleration, with a rate of (ionized) mass loss of the order of $10^{-7} M_{\odot} \mathrm{yr}^{-1}$.

In deriving this mass loss rate value, it is assumed that the ionization fraction does not change over the line emitting region. While this may not be adequate if one considers the wind ionization structure due to the stellar irradiation alone (e.g. Natta et al. 1988), it is a rather acceptable approximation in the present case, given the fact that the lines originate in a compact emitting region close to the source, and that other heating mechanisms have been recognized to be important in maintaining a high degree of ionization in collimated winds (e.g. Shang et al. 2002).

The origin of such an emission in a very compact region close to the source suggests excitation from a stellar wind, more than from a disk-wind unless the emission comes from the inner section of a disk extending down to the source surface. This evidence naturally excludes an origin in MHD disk-winds (e.g. Ferreira \& Pelletier 1993) where the disk is truncated at large distances from the source by the action of a strong magnetic field.

The $\dot{M}_{\text {ion }}$ value derived in our model is moreover large enough to suggest that the gas ionization in the emitting region should be high. An ionization fraction of $\sim 0.1$ would imply an $\dot{M} \sim 10^{-6} M_{\odot} \mathrm{yr}^{-1}$. Values of the order of $10^{-7}-10^{-8} M_{\odot} \mathrm{yr}^{-1}$ have been estimated in T Tauri stars (see e.g. Natta et al. 1988), it is however expected that the efficiency of the mass loss mechanism is higher for younger sources.

Finally the adopted velocity law impies that in the outflowing gas the velocity rapidly increases with the distance from the central source and it is already high $\left(\sim 200 \mathrm{~km} \mathrm{~s}^{-1}\right)$ at about two stellar radii. This velocity trend, which is consistent with magnetically-driven stellar winds (e.g. Lago 1984; Hartmann 1990) as well as with magnetocentrifugal wind models like X-winds (Shu et al. 1994) has been also measured in some T Tauri stars from spectro-astrometric observations of the $\mathrm{H}_{\alpha}$ emission (Takami 2001, 2003).

Our simplified model, in which LTE emission is assumed, necessarily predicts symmetric and centrally peaked emergent line profiles. The P Cygni profiles and blue-shifted absorption components which seem to be a common characteristics of wind models thus far explored, result when large line opacities are coupled with significant deviations from equilibrium in the external expanding region of the wind, where the increasing inefficiency of collisions causes a decrease of the source function. On the other hand, the mere fact that the observed lines appear symmetric and almost Gaussian could be an indication that LTE conditions for the Brackett lines actually hold in the region where they originate. Indeed, Nisini et al. (1995) compared their LTE model with models treating in detail the hydrogen level populations, reaching the conclusions that the LTE assumption is a reasonably good approximation of the Brackett lines, while it may be not totally adequate for the $\mathrm{Pa} \beta$ line. Moreover, the limited $\mathrm{Br} \gamma$ line profile predictions presented by Hartmann (1990) shows that since the upper hydrogen levels reach equilibrium more easily than the Balmer lines, the resulting absorption features are much weaker and the degree of asymmetry significantly reduced. Obviously a more refined model than the one presented here, with a full statistical equilibrium treatment and a more realistic geometry, is needed to better explore the ability of wind models to reproduce the observed Brackett symmetric profiles.

\section{Conclusion}

The analysis of the Brackett decrement in the HH100 IR Class I source has shown that the observed Brackett lines remain optically thick up to a high quantum number. In addition, the profiles of the $\mathrm{Br} \gamma, \mathrm{Br} 12$ and $\mathrm{Br} 13$ lines are fairly symmetric with a tendency of the line $F W H M$ to decrease as the $n$-number increases. On the basis of this evidence we argue that a windlike mechanism, where the gas is accelerating outwards is more suited to reproduce the observed features. A comparison with a very simple model suggests that the emission region should be very compact and close to the stellar surface, a fact favouring a stellar wind or the inner region of a disk wind with a small truncation radius as the natural emission region. The mass loss rate of the ionized gas should be fairly high, of the order of $\dot{M}_{\text {ion }} \sim$ $10^{-7} M_{\odot} \mathrm{yr}^{-1}$, to maintain the lines optically thick. This suggests that the ionization fraction in the emitting gas should not be smaller that $\sim 0.1$ to have a total mass loss rate not exceeding typical values expected from Class I objects.

Our analysis, however, does not solve the problem of how to reproduce the observed symmetrical line profiles of optically thick IR lines, which at present are apparently difficult to model by both wind and accretion models. This probably points to the fact that the real situation is more complicated than described in the simple model presented here. Another caveat to the derived conclusions is the near IR photometric variability of HH100 IR, which shows differences in the $K$ band of more than a magnitude over a period of a few years. Such a variability can also affect the line emission of this source. Indeed, variability not only of HI NIR line fluxes but also of their profiles has been observed in other Class I sources exhibiting photometric variability (Nisini et al. 1994).

Finally, our conclusions are valid for the specific case of HH100IR and as such cannot be generalized to other Class I objects. Indeed, more asymmetric profiles of the $\mathrm{Br} \gamma$ line in similar sources have been succesfully modelled 
by magnetospheric accretion models, such as e.g. WL16 (Muzerolle et al. 1998b). For a better understanding of the origin of the HI lines in Class I sources, a more detailed modelling effort is needed, allowing us to fit in a consistent way both the intensity and profiles of the different observed lines.

Acknowledgements. We thank the anonymous referee for making useful suggestions which helped to clarify some aspects of the paper. This research has made use of NASA's Astrophysics Data System Bibliographic Services and the SIMBAD database, operated at CDS, Strasbourg, France.

\section{References}

Benedettini, M., Nisini, B., Giannini, T., et al. 1998, A\&A, 339, 159 Calvet, N., \& Hartmann, L. 1992, ApJ, 386, 239

Calvet, N., Hartmann, L., \& Hewett, R. 1992, ApJ, 386, 229

Davis, C. J., Ray, T. P., Desroches, L., \& Aspin, C. 2001, MNRAS, 326,524

Edwards, S., Cabrit, S., Strom, S. E., et al. 1987, ApJ, 321, 473

Edwards, S., Hartigan, L., Ghandour, L., \& Andrulis, C. 1994, AJ, 108,1056

Ferreira, J., \& Pelletier, G. 1993, A\&A, 276, 625

Folha, D. F., \& Emerson, J. P. 2001, A\&A, 365, 90

Graham, J. A. 1998, ApJ, 492, 213

Greene, T. P., \& Lada, C. J. 1996, ApJ, 112, 2184

Harju, J., Haikala, L. K., Mattila, K., et al. 1993, A\&A, 278, 569

Hartmann, L., Calvet, N., Avrett, E. H., \& Loeser, R. 1990, ApJ, 349, 168
Lago, M. T. V. T. 1982, MNRAS, 198, 445

Hummer, D. G., \& Storey, P. J. 1987, MNRAS, 224, 801

Marraco, H. G., \& Rydgren, A. E. 1981, AJ, 86, 62

Molinari, S., Liseau, R., \& Lorenzetti, D. 1993, A\&AS, 101, 59

Muzerolle, J., Calvet, N., \& Hartmann, L. 2001, ApJ, 550, 944

Muzerolle, J., Calvet, N., \& Hartmann, L. 1998a, ApJ, 492, 743

Muzerolle, J., Hartmann, L., \& Calvet, N. 1998b, AJ, 116, 2965

Najita, J., Carr, J. S., \& Tokunaga, A. T. 1996, ApJ, 456, 292

Natta, A., Giovanardi, C., \& Palla, F. 1988, ApJ, 332, 921

Nisini, B., Smith, H. A., Fischer, J., \& Geballe, T. R. 1994, A\&A, 290, 463

Nisini, B., Milillo, M., Saraceno, P., \& Vitali, F. 1995, A\&A, 302, 191

Nisini, B., Antoniucci, S., Giannini, T., \& Lorenzetti, D. 2004, in preparation

Reipurth, B., Pedrosa, A., \& Lago, M. T. V. T. 1996, A\&ASS, 120, 229

Rieke, G. H., \& Lebofsky, M. J. 1985, ApJ, 288, 618

Shang, H., Glassgold, A. E., Shu, F. H., \& Lizano, S. 2002, ApJ, 564, 853

Shu, F. H., Najita, J., Ruden, S. P., \& Lizano, S. 1994, ApJ, 429, 797

Storey, P. J., \& Hummer, D. G. 1995, 272, 41

Takami, M., Bailey, J., Gledhill, T. M., Chrysostomou, A., \& Hough, J. H. 2001, MNRAS, 323, 177

Takami, M., Bailey, J., \& Chrysostomou, A. 2003, A\&A, 397, 675

Wilking, B. A., Greene, T. P., Lada, C. J., Meyer, M. R., \& Young, E. T. 1992, ApJ, 397, 520

Whelan, E. T., Ray, T. P., \& Davis, C. J. 2004, A\&A, 417, 247

Whittet, D. C. B., Smith, R. G., Adamson, A. J., et al. 1996, ApJ, 458, 363 